are a general representation of the consolidation problem after finishing of the sedimentation process. In Fig. 2 the upper curves correspond to the greater values of $k / a$

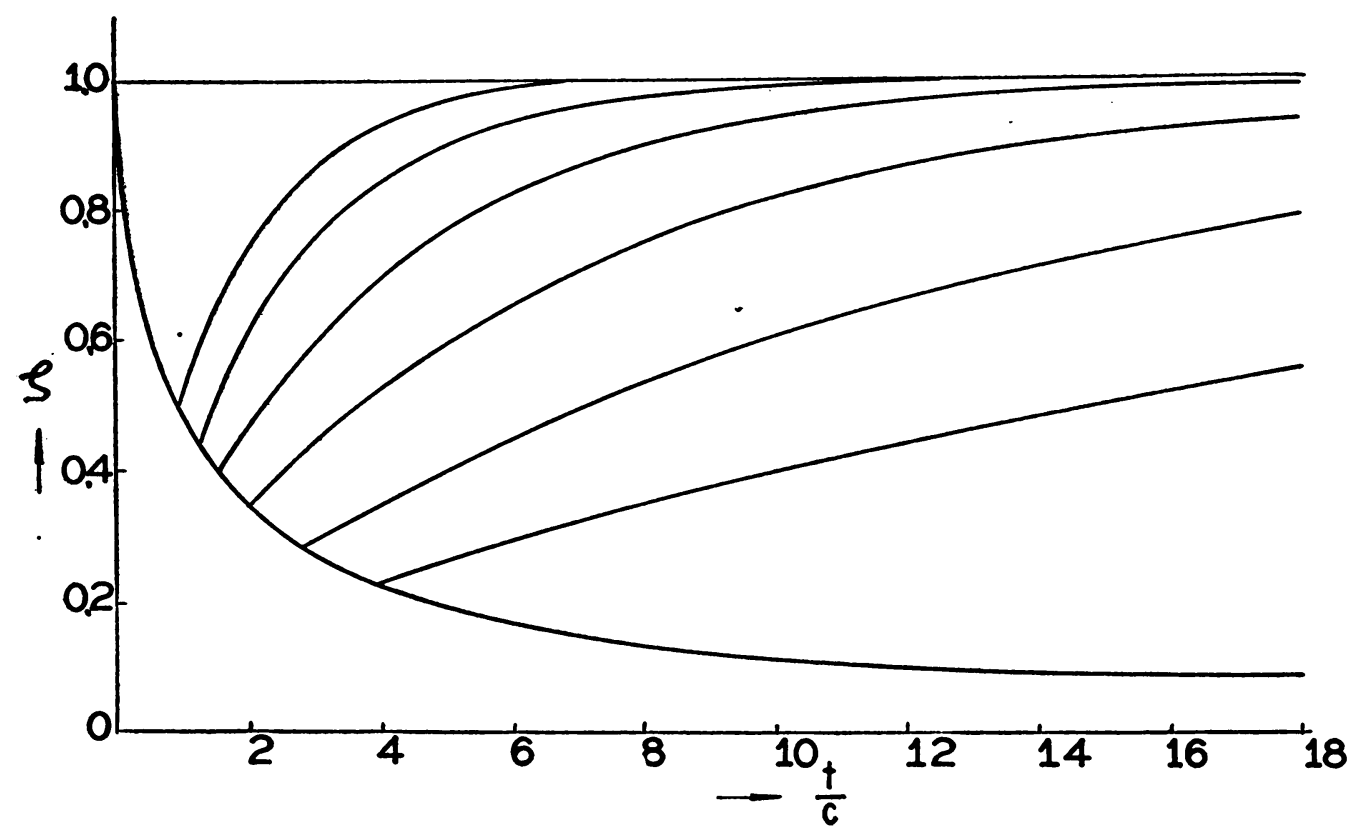

Fig. 2

as valid for sand and other permeable materials, whereas the lower curves correspond to clay and similar earth masses with smaller values of the coefficient of permeability.

\title{
NOTE ON THE PROBLEM OF TWISTING OF A CIRCULAR RING SECTOR*
}

BY ERIC REISSNER (Massachusetts Institute of Technology)

The problem of twisting of a circular ring sector is of some interest in connection with the calculation of stresses and deformations in close-coiled helical springs. To be considered is a ring-sector under the action of two equal and opposite forces $P$ along the axis through the center of the ring and perpendicular to the plane of the ring (Fig. 1). A formulation of the problem and an outline of results by O. Göhner for sectors of solid circular and rectangular cross section may be found on pp. 355-361 in Theory of elasticity by S. Timoshenko.

The purpose of the present note is to obtain explicit results for the twisting of ring sectors of hollow cross sections, with thin walls. Formulas will be obtained which have the same meaning for the present problem as R. Bredt's formulas have for the problem of St. Venant torsion of cylindrical rods.

The problem may be considered as one of the membrane theory of thin shells of

${ }^{*}$ Received Dec. 23, 1948. Work on this note was supported by the Office of Naval Research under Contract N5ori-07834. 


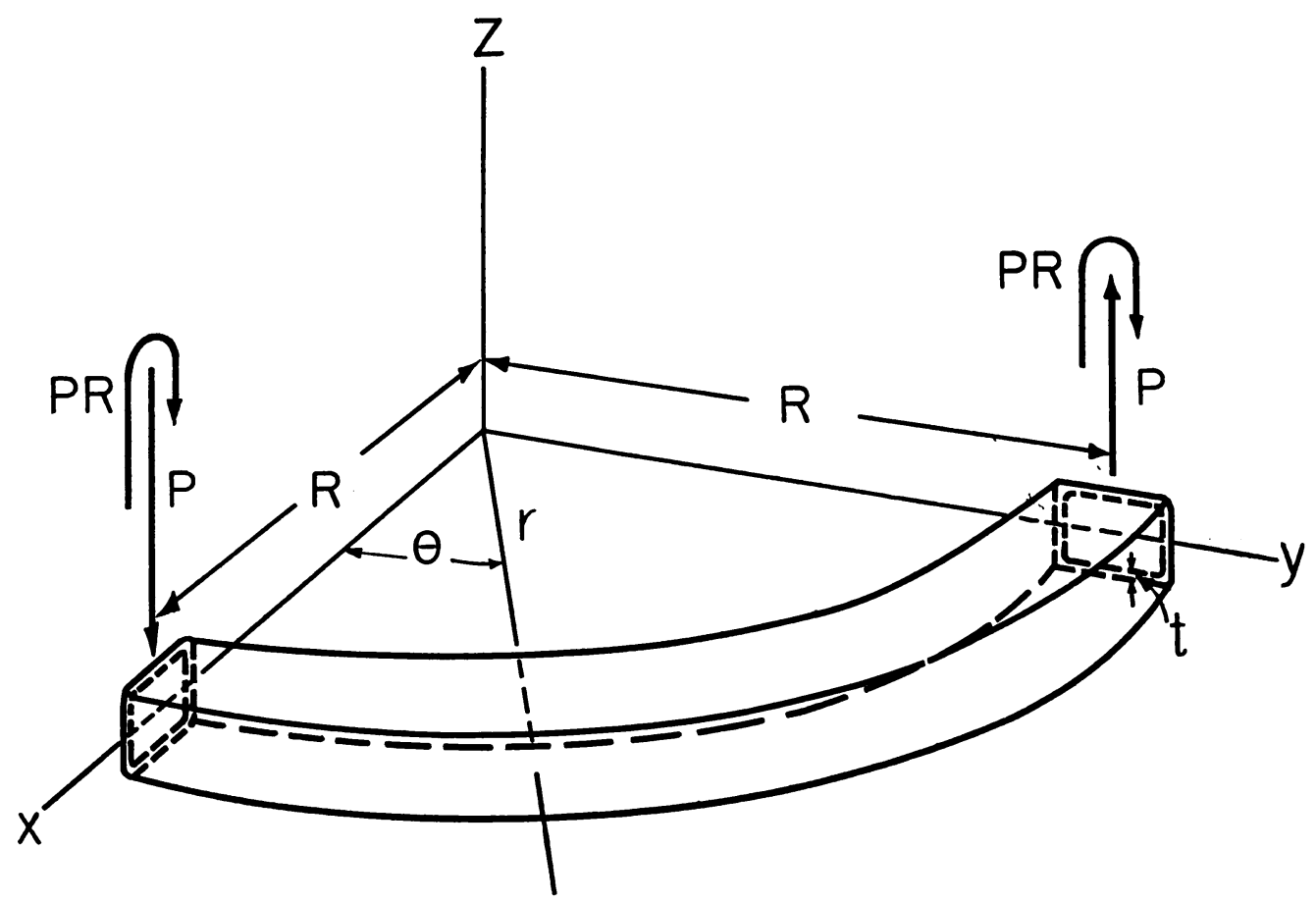

FIG. 1

revolution, with multi-valued expressions for displacements. A direct solution, without recourse to general shell theory may be obtained as follows. The assumptions of the twisting theory of ring sectors are equivalent to requiring that all stress resultants of the membrane theory vanish with the exception of the shear stress resultant $S$ acting

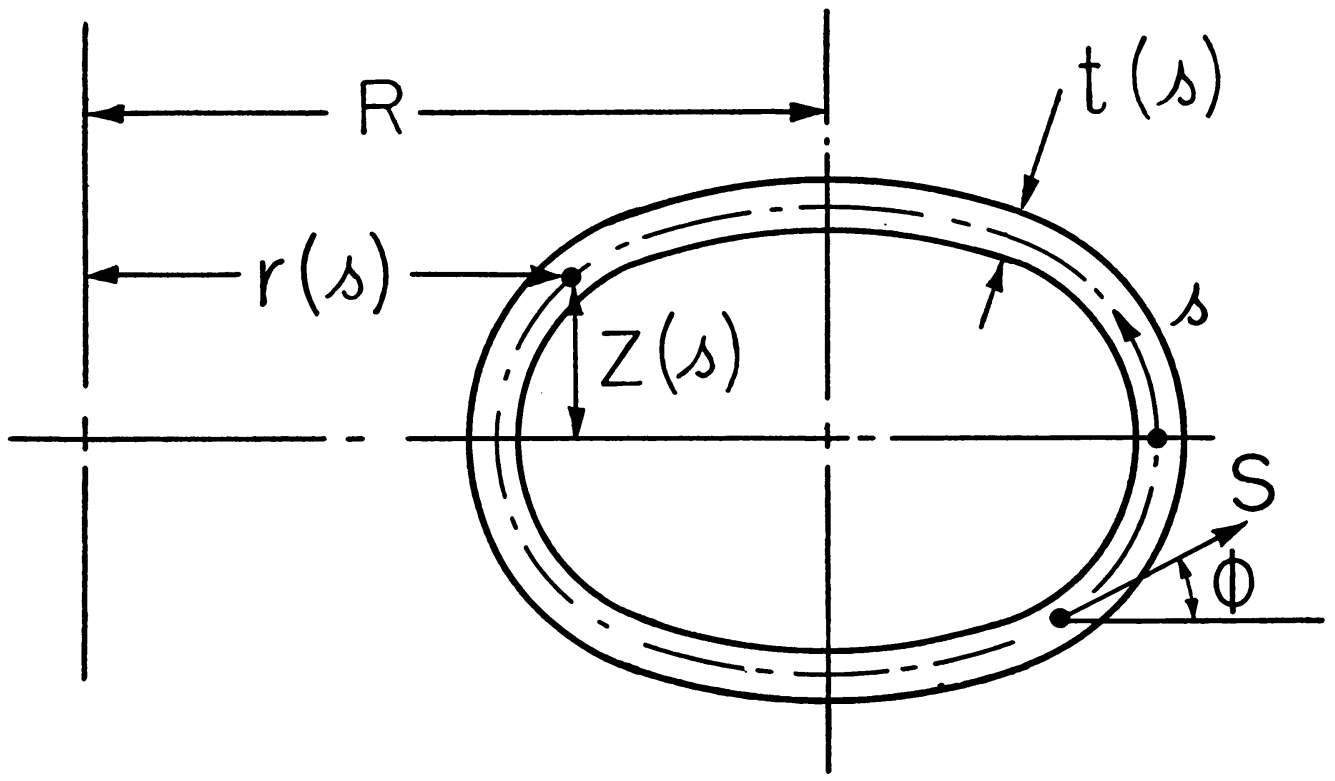

FIg. 2 
over the cross section of the ring sector. (Fig. 2). As all stresses possess rotational symmetry the resultant $S$ satisfies the following equilibrium equation

$$
\frac{d(r S)}{d s}+S \frac{d r}{d s}=0,
$$

where $s$ is the arc length measured along the center line of the tube wall and $r$ is the distance of the points of this line from the axis of the ring sector. From (1) it follows that

$$
r^{2} S=C .
$$

Note that the largest value of the shear stress resultant $S$ occurs at the point nearest the axis. From the relation

$$
P=\oint S \sin \phi d s
$$

the value of the constant $C$ is obtained:

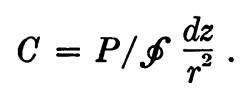

Equation (2) may thus be written in the following form

$$
S=\frac{P}{r^{2} \mathscr{\Phi}\left(1 / r^{2}\right) d z} .
$$

Equation (5) is the counterpart of Bredt's formula $S=T / 2 A$ of torsion theory for the shear stress resultant $S$ in terms of the twisting couple $T$.

For the determination of the deformation of the ring sector we use the stress-strain relation

$$
S=G t \gamma
$$

where $G$ is the modulus of rigidity, $t$ the wall thickness and $\gamma$ the appropriate shearing strain component. In terms of the components of strain in cylindridal coordinates $\gamma_{\theta r}$ and $\gamma_{\theta z}$ we have

$$
\gamma=\gamma_{\theta r} \cos \phi+\gamma_{\theta s} \sin \phi .
$$

In view of the fact that $S$ is the only non-vanishing stress resultant, all other components of strain for cylindrical coordinates vanish,

$$
\epsilon_{r}=\epsilon_{\theta}=\epsilon_{z}=\gamma_{r s}=0 .
$$

Components of displacement in the radial, circumferential and axial directions which are compatible with (8) are*

$$
U=0, \quad V=V(r, z), \quad W=k \theta .
$$

From (9) the remaining components of strain are obtained in the form

$$
\begin{aligned}
& \gamma_{\theta r}=r \frac{\partial}{\partial r}\left(\frac{V}{r}\right)+\frac{1}{r} \frac{\partial U}{\partial \theta}=r \frac{\partial}{\partial r}\left(\frac{V}{r}\right), \\
& \gamma_{\theta z}=\frac{\partial V}{\partial z}+\frac{1}{r} \frac{\partial W}{\partial \theta}=\frac{\partial V}{\partial z}+\frac{k}{r} .
\end{aligned}
$$
sector.

*These expressions hold without any assumption concerning the form of the cross section of the ring 
Combination of (10) and (7) gives

$$
\gamma=r \frac{\partial}{\partial r}\left(\frac{V}{r}\right) \frac{d r}{d s}+\frac{\partial V}{\partial z} \frac{d z}{d s}+\frac{k}{r} \frac{d z}{d s}
$$

or

$$
\frac{\gamma}{r}=\frac{d}{d s}\left(\frac{V}{r}\right)+\frac{k}{r^{2}} \frac{d z}{d s}
$$

In Eq. (12) one takes $\gamma$ in terms of $S$ from Eq. (6) and then integrates (12) over the closed cross section. In view of the fact that $V$ must be a univalued function this leads to the following relation:

$$
\oint \frac{S d s}{G t r}=k \oint \frac{d z}{r^{2}}
$$

or, with $S$ from (5),

$$
\frac{k}{P}=\oint \frac{d s}{G t r^{3}}\left\{\oint \frac{d z}{r^{2}}\right\}^{-2}
$$

where according to (9) the change in length per winding of a spring has a value $2 \pi k$. Equation (14) is the counterpart of the well-known Bredt formula $\theta / T=[\Phi(d s / G t)] / 4 A^{2}$ for the twist-torque ratio for closed thin-walled sections.

Examples. We take for a first example the case of a tube with circular cross section and with uniform wall thickness. The equations of the center line of the tube wall are taken in the form

$$
r=R+a \cos \psi, \quad z=a \sin \psi .
$$

The integrals occurring in Eqs. (5) and (14) become

$$
\begin{aligned}
\oint \frac{d z}{r^{2}} & =\frac{a}{R^{2}} \int_{0}^{2 \pi} \frac{\cos \psi d \psi}{[1+(a / R) \cos \psi]^{2}} \\
& =-\frac{2 \pi}{R} \frac{(a / R)^{2}}{\left[1-(a / R)^{2}\right]^{3 / 2}}
\end{aligned}
$$

and

$$
\begin{aligned}
\oint \frac{d s}{r^{3}} & =\frac{a}{R^{3}} \int_{0}^{2 \pi} \frac{d \psi}{[1+(a / R) \cos \psi]^{3}} \\
& =\frac{\pi a}{R^{3}} \frac{2+(a / R)^{2}}{\left[1-(a / R)^{2}\right]^{5 / 2}} .
\end{aligned}
$$

The ratio (5) between stress resultant $S$ and applied force $P$ is then

$$
\frac{S}{P}=\frac{R}{2 \pi a^{2}} \frac{\left[1-(a / R)^{2}\right]^{3 / 2}}{[1+a / R \cos \psi]^{2}} .
$$

As the ratio $a / R$ tends to zero the tube stress resultant $S$ approaches the value $S=$ $P R / 2 \pi a^{2}=P R / 2 A$ which coincides, as it should, with the value predicted by pure torsion theory for an applied couple of magnitude $P R$. 
The maximum stress occurs when $\cos \psi=-1$ and is of the following magnitude

$$
\begin{aligned}
\frac{S_{\max }}{P} & =\frac{R}{2 \pi a^{2}} \frac{\left[1-(a / R)^{2}\right]^{3 / 2}}{[1-(a / R)]^{2}} \\
& =\frac{R}{2 \pi a^{2}}\left[1+2 \frac{a}{R}+\frac{3}{2}\left(\frac{a}{R}\right)^{2}+\cdots\right] .
\end{aligned}
$$

The deflection-force ratio $k / P$ as given by Eq. (14) becomes

$$
\begin{aligned}
\frac{k}{P} & =\frac{1}{G t} \frac{R^{3}}{2 \pi a^{3}}\left[1-\left(\frac{a}{R}\right)^{2}\right]^{1 / 2}\left[1+\frac{1}{2}\left(\frac{a}{R}\right)^{2}\right] \\
& =\frac{1}{G t} \frac{R^{3}}{2 \pi a^{3}}\left[1-\frac{3}{8}\left(\frac{a}{R}\right)^{4}-\frac{1}{8}\left(\frac{a}{R}\right)^{\circ}-\cdots\right] .
\end{aligned}
$$

The absence of a term of the form $(a / R)^{2}$ inside the bracket indicates that the influence of the factor in brackets is quite small in all but extreme cases.

As a second example we consider a tube with rectangular cross section and uniform thickness. We designate by $R$ the distance of the center of the cross section from the axis, by $2 a$ the width of the tube and by $2 b$ the height of the tube. We find that

$$
\Phi \frac{d z}{r^{2}}=-\frac{8 a b}{R^{3}}\left[\frac{1}{\left.1-(a / R)^{2}\right]^{2}}\right.
$$

and

$$
\mathscr{S} \frac{d s}{r^{3}}=\frac{4(a+b)}{R^{3}} \frac{1+(3 b-a)(a / R)^{2} /(b+a)}{\left[1-(a / R)^{2}\right]^{3}}
$$

From (21) and (5) the maximum value of the stress resultant $S$ follows in the form

$$
\begin{aligned}
\frac{S_{\max }}{P} & =\frac{R}{8 a b} \frac{\left[1-(a / R)^{2}\right]^{2}}{[1-(a / R)]^{2}} \\
& =\frac{R}{8 a b}\left[1+2 \frac{a}{R}+\left(\frac{a}{R}\right)^{2}+\cdots\right] .
\end{aligned}
$$

It is noteworthy that the factor in brackets in (23) is independent of the height $2 b$ of the tube cross section, and that this factor is somewhat smaller than the corresponding factor of Eq. (19) for the circular tube.

Combination of (14), (21) and (22) gives for the deflection-force ratio of the rectangular tube the following result

$$
\begin{aligned}
\frac{k}{P} & =\frac{1}{G t} \frac{(a+b) R^{3}}{16 a^{2} b^{2}}\left[1-\left(\frac{a}{R}\right)^{2}\right]\left[1+\frac{3 b-a}{b+a}\left(\frac{a}{R}\right)^{2}\right] \\
& =\frac{1}{G t} \frac{(a+b) R^{3}}{16 a^{2} b^{2}}\left[1+2 \frac{b-a}{b+a}\left(\frac{a}{R}\right)^{2}-\frac{3 b-a}{b+a}\left(\frac{a}{R}\right)^{4}\right] .
\end{aligned}
$$

A comparison with the corresponding formula (20) shows that the square tube shares with the circular tube the property that no terms with $(a / R)^{2}$ occur within the last bracket. In contrast to this, terms with $(a / R)^{2}$ do occur whenever one of the sides of 
the rectangle is longer than the other. It may be of interest to list the following special cases. Denoting the factor in brackets by $1+\delta$, we find that when

$$
a \ll b, \quad 1+\delta \sim 1+2(a / R)^{2}-3(a / R)^{4},
$$

and when

$$
b \ll a, \quad 1+\delta \sim 1-2(a / R)^{2}+(a / R)^{4} .
$$

It may be noted when $a \ll b$ then $\delta$ assumes a maximum value of $5 / 27$ for $(a / R)^{2}=$ $1 / 3$, whereas when $b \ll a$ then $\delta$ is always negative.

CORRECTIONS* TO THE PAPER

ON A CLASS OF SINGULAR INTEGRAL EQUATIONS OCCURRING IN PHYSICS

Quarterly of Applied Mathematics 6, 443-448 (1949)

Br H. P. THIELMAN (Iowa State College)

The limits on the integral in Eq. (B), p. 445 have been omitted. They should have been indicated as 0 and $\infty$.

Equation (a) of Theorem I, p. 445 should read $k f(0)-f^{\prime}(0)=0$ and not $k f(0)-$ $f^{\prime \prime}(0)=0$ as stated. It should have been stated that $f^{\prime \prime}(x)$ in Theorem I, and $f^{\text {iv }}(x)$ in Theorem II are assumed to be of order $o\left(e^{k x}\right)$ as $x$ goes to infinity.

*Received June 6, 1949.

\section{BOOK REVIEWS}

Proceedings of a symposium on large-scale digital calculating machinery. Jointly sponsored by the Navy Department Bureau of Ordnance and Harvard University at the Computation Laboratory. Harvard University Press, Cambridge, Massachusetts, 1948. xxix +302 pp. $\$ 10.00$.

This is a collection of papers and discussions of papers presented at a symposium on large-scale digital computing machinery held at Harvard University on January 7-10, 1947. The meeting was sponsored jointly by the Navy Department Bureau of Ordnance and Harvard University. The book contains numerous photographs and drawings. The technical addresses covered eight sessions dealing with the general topics of "Existing Calculating Machines", "The Logic of Large Scale Calculating Machinery", "Storage Devices", "Numerical Methods and Suggested Problems for Solution", "Sequencing, Coding and Problem Preparation", "Input and Output Devices", "Conclusions and Open Discussion". The state of the art seems to have been well surveyed. 